\title{
A High Dose of Fentanyl May Accelerate the Onset of Acute Postoperative Pain
}

\author{
Anna Rupniewska-Ladyko (iD ${ }^{1,{ }^{*}}$ and Malgorzata Malec-Milewska ${ }^{1}$ \\ ${ }^{1}$ Department of Anesthesiology and Intensive Care, Centre of Postgraduate Medical Education, Warsaw, Poland \\ "Corresponding author: Department of Anesthesiology and Intensive Care, CMKP ul. Czerniakowska, 231 00-416, Warsaw Poland. Email: aavr@interia.pl
}

Received 2019 May 26; Revised 2019 July 17; Accepted 2019 August 16.

\begin{abstract}
Fentanyl is a popular intraoperative analgesic linked with the development of opioid tolerance (OT) or opioid-induced hyperalgesia (OIH). The development of OIH or OT may lead to several issues such as delayed healing after surgery and timely discharge. Moreover, it causes discomfort in patients with higher pain scores, greater use of analgesics, and other associated side effects. The current study aimed at determining whether the amount of intraoperatively administered fentanyl affects the onset of acute postoperative pain. The current retrospective study was conducted on 56 patients undergoing laparoscopic sleeve gastrectomy. In patients receiving relatively large doses of fentanyl intraoperatively (over $3 \mu \mathrm{g} / \mathrm{kg}$ body weight), the onset of acute postoperative pain accelerated significantly, in comparison with the ones receiving lower doses.
\end{abstract}

Keywords: Fentanyl, Opioid Tolerance, Opioid-Induced Hyperalgesia, Postoperative Pain, Perioperative Period, General Anesthesia

\section{Background}

Fentanyl is increasingly employed in operations with respect to providing perioperative analgesia as well as stable hemodynamics; nonetheless, since fentanyl is linked with the development of opioid tolerance (OT) (increased dose needed for analgesia) and opioid-induced hyperalgesia (OIH) (paradoxical increase in pain with opioid administration), it can contribute to both poorly controlled pain and dose escalation (1-3). It is reported that high intraoperative doses of opioid may increase postoperative pain scores and acute opioid consumption, and also induce significant nociceptive threshold changes (4).

The current study aimed at evaluating the effects of various doses of intraoperatively administered fentanyl (used in sleeve-gastrectomy) on the onset of acute postoperative pain.

The obtained results showed that the development of fentanyl OT and OIH was a clinically significant phenomenon, requiring further research.

The patient files registered from 2013 to 2015 were examined. It was decided to choose the cases of patients that underwent laparoscopic sleeve gastrectomy, due to the homogeneous surgical technique and the same anesthesia scheme involved.

\section{Objectives}

The current retrospective study enrolled 56 patients. The effect of the total amount of intraoperatively administered fentanyl on the time to onset of PCA (patientcontrolled analgesia) was statistically analyzed in the patients.

\section{Methods}

After obtaining the ethical approval from Ethical Committee Centre of Postgraduate Medical Education in Warsaw, Poland (Chairperson Prof. J. Reguła), data on anesthetic and postoperative observations of laparoscopic sleeve gastrectomy (performed from 2013 to 2015) were retrospectively obtained from hospital archives.

The current retrospective study enrolled 56 patients (45 males and 11 females) with ASAPS (the American Society for Aesthetic Plastic Surgery) classes 1 and 2 and body mass index (BMI) $47 \mathrm{~kg} / \mathrm{m}^{2}$, who underwent general anesthesia maintained with air, $\mathrm{O}_{2}$, desflurane, rocuronium bromide, fentanyl, and sugammadex. Exclusion criteria were: receiving opioid-sparing pharmacological adjuvants, the occurrence of intraoperative circulatory-respiratory disorders, and employment of other methods of anesthesia or postoperative pain treatment. 
The pain treatment continued using PCA with intravenous oxycodone.

CONSORT guidelines were applied in the current study. The characteristics of the collected materials were described using standard descriptive statistics. The level of significance was 0.05; confidence interval (CI) for intraand inter-group mean differences was 95\%. Additionally, statistical analysis was performed in the R computing environment (5).

\section{Results}

The mean operation time was 97 minutes and the total dose of intraoperatively administered fentanyl was 0.3-0.7 mg. No significant differences were observed in the duration of surgery, heart rate, blood pressure, oxygen saturation, capnometry, anesthesia, or BMI between the groups. The mean score of postoperative pain based on numeric rating scale (NRS) was 2 , and postoperative follow-up was 12 hours.

Patients receiving a higher amount of fentanyl during surgery reported the onset of postoperative pain a lot earlier and required an analgesic.

Administration of each dose of fentanyl $(0.1 \mathrm{mg})$ during general anesthesia ( $>3 \mu \mathrm{g} / \mathrm{kg}$ ) accelerated the onset of acute postoperative pain by 14.7 minutes $(\mathrm{P}<0.005)$ (Figure 1).

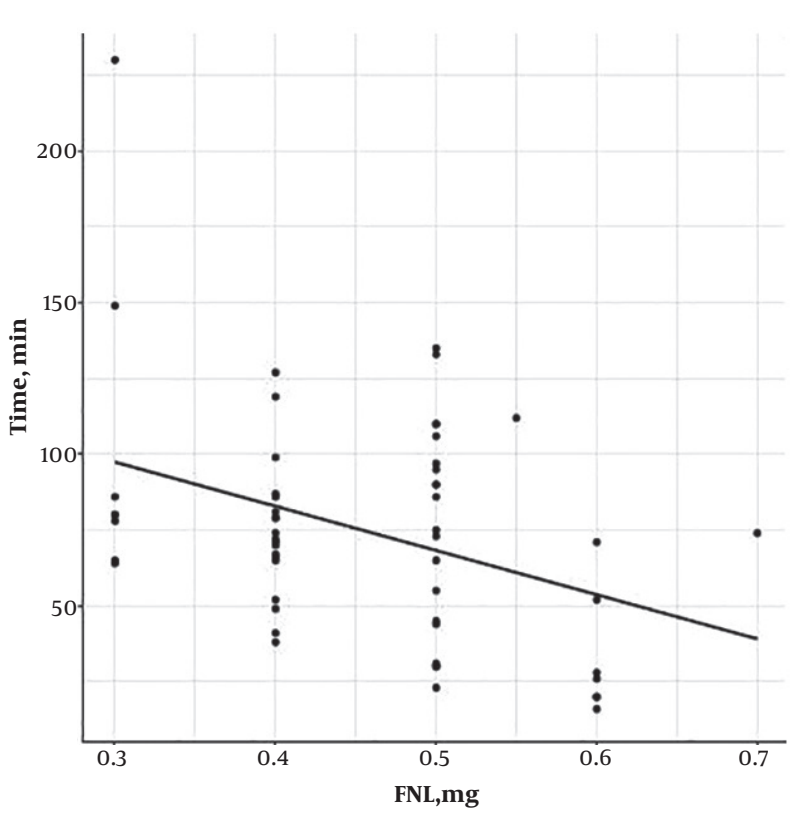

Figure 1. The relationship between the total dose of intraoperatively administered fentanyl (FNL) and time (min) to analgesic request in the studied patients

\section{Discussion}

OT and OIH are comprehensively discussed in medical literature, mostly in patients with chronic opioid use (6). In addition, they are frequently mentioned in relation to remifentanil infusions $(1,6)$, while the literature on $\mathrm{OIH}$ or OT caused by intraoperative fentanyl use is scarce.

Until recently, it was acknowledged that the mode of fentanyl dosing has no clinical consequences other than sedation and ventilatory depression. However, it is now recognized that OT may be induced by the administration of fentanyl, even for a period as short as the duration of a surgery $(3,6)$. Indeed, it entails the increased use of pain medications postoperatively, with all their possible side effects. The results of the current study indicated a correlation between high doses of fentanyl administered intraoperatively and the early onset of pain along with increased need for opioid analgesics after surgery.

Observations of other studies indicated that in order to reduce intra-operatively administered opioid doses, multimodal analgesia or adjuvant drugs may be helpful $(7,8)$. In order to achieve adequate analgesia, adjuvant drugs including N-methyl-D-aspartate (NMDA) receptor inhibitors such as ketamine, magnesium, dexmedetomidine, or gabapentin can be suggested, as their potentials for pain reduction and OIH/OT prevention is well documented (8-10). Similarly, opioid-free anesthesia appears to be the appropriate option in relation to fentanyl-related OIH/OT.

However, the current study had a few limitations. The study population was relatively small with higher number of female patients (most patients undergoing sleeve gastrectomy are female), and all of them had obesity. Nevertheless, the results corresponded with previously reported findings and pointed to an issue pertaining to the minimization of intraoperative fentanyl use $(2,11)$. Other studies are warranted to further investigate OIH/OT in patients undergoing surgery and receiving fentanyl as a part of general anesthesia.

\subsection{Conclusions}

Patients receiving a higher amount of fentanyl during surgery reported the onset of postoperative pain a lot earlier, requiring an analgesic.

\section{Acknowledgments}

I wish to extend my thanks to Dr. Marek Rupniewski, Warsaw University of Technology, Department of Electronics and Information Technology, Institute of Electronic Systems, Warsaw, Poland for helping me in the statistical analysis of the data, for my research work. 


\section{Footnotes}

Conflict of Interests: The authors declared no conflict of interest.

Ethical Approval: The study protocol was approved by the Ethical Committee Centre of Postgraduate Medical Education in Warsaw, Poland (Chairperson Prof. J. Reguła) on 13 February 2013.

Funding/Support: The study received no financial support for the research, authorship, and/or publication of the article; particularly, any financial agreements they may have with pharmaceutical or biomedical firms whose products are pertinent to the subject matter dealt with in the manuscript.

\section{References}

1. Santonocito C, Noto A, Crimi C, Sanfilippo F. Remifentanil-induced postoperative hyperalgesia: Current perspectives on mechanisms and therapeutic strategies. Local Reg Anesth. 2018;11:15-23. doi: 10.2147/LRA.S143618. [PubMed: 29670398]. [PubMed Central: PMC5898588].

2. Colvin LA, Bull F, Hales TG. Perioperative opioid analgesia-when is enough too much? A review of opioid-induced tolerance and hyperalgesia. Lancet. 2019;393(10180):1558-68. doi: 10.1016/S01406736(19)30430-1. [PubMed: 30983591].

3. Celerier E, Rivat C, Jun Y, Laulin JP, Larcher A, Reynier P, et al. Longlasting hyperalgesia induced by fentanyl in rats: Preventive effect of ketamine. Anesthesiology. 2000;92(2):465-72. doi: 10.1097/00000542200002000-00029. [PubMed:10691234].

4. Weber L, Yeomans DC, Tzabazis A. Opioid-induced hyperalgesia in clinical anesthesia practice: What has remained from theoretical concepts and experimental studies? Curr Opin Anaesthesiol. 2017;30(4):458-65. doi: 10.1097/ACO.0000000000000485. [PubMed: 28590258].

5. R Core Team. R: A language and environment for statistical computing. Vienna, Austria: R Foundation for Statistical Computing; 2013.

6. Xuerong Y, Yuguang H, Xia J, Hailan W. Ketamine and lornoxicam for preventing a fentanyl-induced increase in postoperative morphine requirement. Anesth Analg. 2008;107(6):2032-7. doi: 10.1213/ane.ob013e3181888061. [PubMed:19020155].

7. Macfater H, Xia W, Srinivasa S, Hill AG, Van De Velde M, Joshi GP, et al. Evidence-based management of postoperative pain in adults undergoing laparoscopic sleeve gastrectomy. World J Surg. 2019;43(6):157180. doi: 10.1007/s00268-019-04934-y. [PubMed:30719556].

8. Qiu H, Sun Z, Shadhiya F, Arulthas R, Priya GV, Christopher P, et al. The influence of dexmedetomidine on remifentanil-induced hyperalgesia and the sex differences. Exp Ther Med. 2018;16(4):3596-602. doi: 10.3892/etm.2018.6641. [PubMed: 30233714]. [PubMed Central: PMC6143892]

9. Leal PC, Salomao R, Brunialti MK, Sakata RK. Evaluation of the effect of ketamine on remifentanil-induced hyperalgesia: A double-blind, randomized study. J Clin Anesth. 2015;27(4):331-7. doi: 10.1016/j.jclinane.2015.02.002. [PubMed: 25910532].

10. Song JW, Lee YW, Yoon KB, Park SJ, Shim YH. Magnesium sulfate prevents remifentanil-induced postoperative hyperalgesia in patients undergoing thyroidectomy. Anesth Analg. 2011;113(2):390-7. doi: 10.1213/ANE.ob013e31821d72bc. [PubMed: 21596876].

11. Chia YY, Liu K, Wang JJ, Kuo MC, Ho ST. Intraoperative high dose fentanyl induces postoperative fentanyl tolerance. Can J Anaesth. 1999;46(9):872-7. doi: 10.1007/BF03012978. [PubMed: 10490157]. 